\title{
Legal restrictions block NASA 'institutes'
}

Washington. A year-long effort to establish privatized 'science institutes' at several US space agency field centres appears to have foundered because of legal restrictions on government employees transferring to the private sector.

The National Aeronautics and Space Administration (NASA) had asked for language to be included in its 1997 authorization bill lifting post-employment restrictions on government scientists who might want to convert to contractor status under the institute plan - particularly in relation to their ability to work on contracts they had once managed, and to keep their retirement pay and other benefits.

But the Office of Government Ethics and the Office of Personnel Management both opposed exempting NASA from rules applied to other agencies, and the language was deleted from the authorization bill. As a result, Daniel Goldin, the agency's administrator, has told centre directors in a letter that "we will discontinue our efforts to establish science institutes as previously planned at the Ames Research Center [in California] and the Lewis Research Center [in Cleveland, Ohio]." NASA science managers had maintained that, without 'legislative relief', the science institutes plan might not be viable.

An institute for space biomedical research at the Johnson Space Center in Texas will, however, continue as planned, as it is not expected to draw heavily on government scientists for staff. NASA will be seeking partners for the biomedical institute later this summer. Other proposed institutes at the agency's Langley and Marshall centres were effectively shelved last year because of a lack of clear definition.

Goldin's decision appears to end the troubled saga of the science institutes, which suffered from a vagueness of purpose and a lack of interest among potential university sponsors (see Nature 380, 7; 1996). France Cordova, NASA's chief scientist, says that any further efforts to strengthen the agency's ties with the outside research community will now fall to each individual centre. In his letter to centre directors, Goldin said only that "[w]e will consider other center options that will continue to enhance the quality of the science at the respective field installations".

Exactly what those options are is not clear. William Berry, head of Ames' space directorate, says there is some relief among staff scientists now that uncertainty about the fate of the institutes is over. Rather than create one large umbrella institute, he says, Ames will now work with local universities to set up "new relationships" in specific disciplines, and perhaps consider creating several small institutes.

Berry says that one university has already shown interest in establishing a new astrobiology department, which could collaborate regularly with Ames staff. The Ames science directorate plans a conference this autumn to stimulate additional relationships with outside researchers.

In the long run, Berry expects work on the space station and on NASA's proposed 'origins' programme - which covers disciplines ranging from cosmology to the search for extraterrestrial life - to provide new lines of business for space researchers. But the space directorate faces a funding cut of 20 per cent in the next several years, which suggests that some of the current Ames work force will have to go.

One research task that might be "outsourced", says Berry, involves the large animal centrifuge designed for life sciences research on the space station. He says that he can envisage centrifuge research being devolved to a privatized institute similar to - but much smaller than - the Space Telescope Science Institute in Baltimore, Maryland.

But even if such mini-institutes are established, current conflict-of-interest laws will prevent government scientists from working there. The same restrictions will apply to the Lewis centre's microgravity research programme, which was another likely candidate for privatization. As a result, NASA staff scientists are now facing the same uncertain future that they faced more than a year ago, when the institutes were first proposed.

\section{Rapid response team urged for space station research}

Washington. The research plans of the US National Aeronautics and Space Administration (NASA) and its partners for the international space station neglect engineering and technology research that could help reduce its operational costs and improve its performance, according to the National Research Council (NRC).

But a report published last week by the NRC, the operating arm of the National Academy of Sciences, says that it is not too late to add more experiments to the line-up - provided that NASA quickly forms a 'rapid response' team to suggest modifications to the station before it begins construction next year.

The NRC panel was chaired by David Bodde of the Midwest Research Institute in Kansas City, Missouri. It says that the station could still be a valuable platform for in-space studies of electric-power generation, robotics, propulsion, life support and several other areas of technology - not least of which is the behaviour of large rigid structures in space.

But as plans for the station have been scaled back in recent years, many such experiments have been dropped. The report

\section{IMAGE UNAVAILABLE FOR COPYRIGHT REASONS}

\section{Testing: time for NASA to add experiments?}

lists nearly a dozen 'disappearing technologies', including advanced 'solar dynamic' power generation and closed-loop environmental-control systems that would recycle water and oxygen. Many of these had been studied in detail before NASA dropped them from the plan, and the panel believes they could be put back in.

Other suggestions are that NASA should develop a 'road map' for engineering and technology research, ensure that experiment interfaces on the station are similar to those

$\frac{\pi}{z}$ in laboratories on the ground, and investigate the feasibility of placing instruments on the station to help monitor its structural dynamics and the space environment.

NASA should also involve industry and academic institutions in selecting engineering and technology experiments, says the panel. One of the most controversial suggestions - and probably the least likely to be followed - is that the space agency should auction off perhaps 15 per cent of station resources to commercial researchers, rather than try to decide itself what work has commercial potential.

Many of the criticisms - such as the lack of common interfaces and the difficulty in proposing technology experiments - have been raised by other panels in the past. Indeed, some committee members say NASA is following its usual tendency of focusing on getting new hardware into space rather than on how it will be used.

Nevertheless, Daniel Goldin, the administrator of the agency, was enthusiastic about the report when briefed on its proposals last week, and may soon appoint a task force to look into its recommendations.

T. R. 